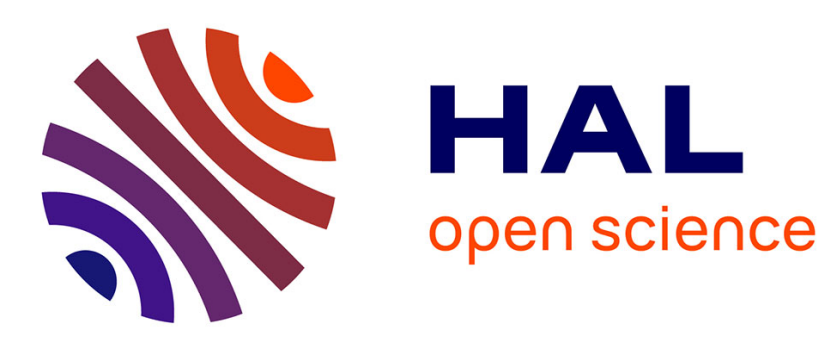

\title{
Sur l'inégalité de Turán-Kubilius friable
}

Régis de La Bretèche, Gérald Tenenbaum

\section{To cite this version:}

Régis de La Bretèche, Gérald Tenenbaum. Sur l'inégalité de Turán-Kubilius friable. Journal of the London Mathematical Society, 2016, 93 (1), pp.175-193. 10.1112/jlms/jdv051 . hal-01281714

\section{HAL Id: hal-01281714 https://hal.science/hal-01281714}

Submitted on 2 Mar 2016

HAL is a multi-disciplinary open access archive for the deposit and dissemination of scientific research documents, whether they are published or not. The documents may come from teaching and research institutions in France or abroad, or from public or private research centers.
L'archive ouverte pluridisciplinaire HAL, est destinée au dépôt et à la diffusion de documents scientifiques de niveau recherche, publiés ou non, émanant des établissements d'enseignement et de recherche français ou étrangers, des laboratoires publics ou privés. 


\title{
Sur l'inégalité de Turán-Kubilius friable
}

\author{
R. de la Bretèche \& G. Tenenbaum
}

\begin{abstract}
We obtain a new form, uniform with respect to all parameters, of the friable (i.e. relevant to integers free of large prime factors) Turán-Kubilius inequality, comparing the empirical variance of an additive arithmetical function with friable support to that of its probabilistic model. Several applications are developed, significantly improving on previously known results for small values of the friability parameter.
\end{abstract}

Keywords: Friable integers, additive functions, Kubilius model, Turán-Kubilius inequality.

Résumé. Nous établissons une version uniforme, relativement à tous les paramètres, de l'inégalité de Turán-Kubilius friable (i.e. relative aux entiers sans grand facteur premier), comparant la variance empirique d'une fonction arithmétique additive à support friable à celle de son modèle probabiliste. Nous développons plusieurs applications améliorant significativement des résultats antérieurement connus pour les petites valeurs du paramètre de friabilité.

Mots clefs : Entiers friables, fonctions additives, modèle de Kubilius, inégalité de TuránKubilius.

\section{Introduction et énoncé}

La théorie des entiers friables, ou sans grand facteur premier, occupe une place grandissante au sein de théorie analytique et probabiliste des nombres. L'un des outils les plus productifs en termes d'applications est l'extension à ce cadre de l'inégalité de TuránKubilius. Abordée dans [1], puis [10], [11], cette étude a été poursuivie et uniformisée dans [3], [8], [6], [4]. Nous nous proposons ici d'apporter une réponse à l'une des questions fondamentales restant en suspens et de développer quelques conséquences.

Soit $\mathbb{A}$ la classe des fonctions arithmétiques additives complexes. Pour $x \geqslant y \geqslant 2$, nous désignons par $S(x, y)$ l'ensemble des entiers $y$-friables n'excédant pas $x,^{(1)}$ et par $\alpha=\alpha(x, y)$ le point-selle associé à l'intégrale de Perron pour $\Psi(x, y):=|S(x, y)|$, défini par l'équation transcendante

$$
\sum_{p \leqslant y} \frac{\log p}{p^{\alpha}-1}=\log x .
$$

Dans tout ce travail, nous réservons la lettre $p$ pour désigner un nombre premier.

Dans [3], nous avons proposé, pour chaque fonction $f$ de $\mathbb{A}$, un modèle probabiliste $Z_{f}=Z_{f, x, y}$ de la restriction de $f$ à $S(x, y)$, défini par la formule

$$
Z_{f}:=\sum_{p \leqslant y} \xi_{p}
$$

où $\xi_{p}=\xi_{p}(f)$ est une variable aléatoire géométrique telle que

$$
\mathbb{P}\left(\xi_{p}=f\left(p^{\nu}\right)\right)=g_{p}(\alpha) / p^{\nu \alpha} \quad(\nu \in \mathbb{N}),
$$

les $\xi_{p}$ étant supposées indépendantes, avec la convention que $f\left(p^{\nu}\right)=0$ si $p^{\nu}>x$. Ici et dans la suite, nous utilisons la notation

$$
g_{m}(s):=\prod_{p \mid m}\left(1-1 / p^{s}\right) \quad\left(m \in \mathbb{N}^{*}, s \in \mathbb{C}\right) .
$$

1. Autrement dit l'ensemble de tous les entiers naturels $n \leqslant x$ dont le plus grand facteur premier est $\leqslant y$. 
Par convention, si plusieurs valeurs (éventuellement en nombre infini) $f\left(p^{\nu}\right)$ sont égales, la probabilité correspondante figurant au membre de gauche de $(1 \cdot 2)$ est définie comme la somme des quantités apparaissant au membre de droite.

Comme dans [3], nous définissons la variance semi-empirique $V_{f}$ de $f$ sur $S(x, y)$ par la formule

$$
V_{f}=V_{f}(x, y):=\frac{1}{\Psi(x, y)} \sum_{n \in S(x, y)}\left|f(n)-\mathbb{E}\left(Z_{f}\right)\right|^{2}
$$

avec donc

$$
\mathbb{E}\left(Z_{f}\right)=\sum_{p^{\nu} \in S(x, y)} \frac{g_{p}(\alpha) f\left(p^{\nu}\right)}{p^{\nu \alpha}}
$$

Dans son acception la plus forte, l'inégalité de Turán-Kubilius friable consiste en une majoration de $V_{f}(x, y)$ par un multiple constant de $\mathbb{V}\left(Z_{f}\right)$. Nous avons

$$
\mathbb{V}\left(Z_{f}\right):=\sum_{p^{\nu} \in S(x, y)} \frac{g_{p}(\alpha)}{p^{\nu \alpha}}\left|f\left(p^{\nu}\right)\right|^{2}-\sum_{p \leqslant y}\left|\sum_{1 \leqslant \nu \leqslant \nu_{p}} \frac{g_{p}(\alpha) f\left(p^{\nu}\right)}{p^{\nu \alpha}}\right|^{2},
$$

où nous avons posé $\nu_{p}=\nu_{p}(x):=\lfloor(\log x) / \log p\rfloor(p \leqslant y)$.

Les résultats de [3] et [4], fournissent la majoration souhaitée

$$
V_{f} \ll \mathbb{V}\left(Z_{f}\right)
$$

dans le domaine défini par la condition $\sqrt{\log x} \log _{2} x \leqslant y \leqslant x$. De plus, le théorème 1.1 de [3] implique (1.6) lorsque les variables aléatoires $\xi_{p}$ sont centrées, autrement dit lorsque

$$
\sum_{1 \leqslant \nu \leqslant \nu_{p}} \frac{g_{p}(\alpha) f\left(p^{\nu}\right)}{p^{\nu \alpha}}=0 \quad(p \in \mathcal{P}) .
$$

Ici et dans la suite, $\mathcal{P}$ désigne l'ensemble des nombres premiers.

Le présent travail est consacré à l'extension du domaine de validité de (1.6). Nous obtenons le résultat suivant.

Théorème 1.1. La majoration (1.6) est valable uniformément pour $f \in \mathbb{A}$ et $x \geqslant y \geqslant 2$.

Posons

$$
\begin{aligned}
& B_{f}^{2}=B_{f}(x, y)^{2}:=\sum_{p^{\nu} \in S(x, y)} \frac{g_{p}(\alpha)}{p^{\nu \alpha}}\left|f\left(p^{\nu}\right)\right|^{2}, \\
& \left(B_{f}^{-}\right)^{2}=B_{f}^{-}(x, y)^{2}:=\sum_{p^{\nu} \in S(x, y)} \frac{g_{p}(\alpha)^{2}}{p^{\nu \alpha}}\left|f\left(p^{\nu}\right)\right|^{2} .
\end{aligned}
$$

L'inégalité de Cauchy-Schwarz permet d'écrire

$$
\left(1-2^{-\alpha}\right) B_{f}^{2} \leqslant\left(B_{f}^{-}\right)^{2} \leqslant \mathbb{V}\left(Z_{f}\right) \leqslant B_{f}^{2} .
$$

Notre approche est fondée sur le fait que l'inégalité

$$
V_{f}(x, y) \ll B_{f}(x, y)^{2} \quad(f \in \mathbb{A}, x \geqslant y \geqslant 2),
$$


établie dans [3] en toute généralité, permet de ramener la preuve de (1.6) au cas d'une fonction fortement additive. Sous l'hypothèse $y \ll \log x$, une estimation générale, prouvée dans [2], du comportement local de $\Psi(x, y)$ fournit alors rapidement l'estimation souhaitée.

Il est à noter que le modèle (1.2) comporte un biais systématique, dû au fait que les probabilités ainsi définies ne sont pas nulles lorsque $p^{\nu}>x$. Ce phénomène est particulièrement évident lorsque $f$ est fortement additive : notant

$$
w_{p}=w_{p}(x, y):=p^{-\alpha \nu_{p}},
$$

nous avons alors

$$
\mathbb{P}\left(\xi_{p}=f(p)\right)=\frac{1-w_{p}}{p^{\alpha}}
$$

alors que (voir en particulier [3] ou (3.4) infra) l'approximation au premier ordre de $\Psi(x / p, y) / \Psi(x, y)$ est $1 / p^{\alpha}$. Nous avons - cf., par exemple, [2], formule (3.4) -

$$
x^{-\alpha} \leqslant w_{p} \leqslant \min \left\{(y / x)^{\alpha}, x^{-\alpha / 2}\right\}, \quad(x y)^{-\alpha} \leqslant w_{p} / p^{\alpha} \leqslant x^{-\alpha},
$$

de sorte que le biais n'a qu'une influence marginale dès que $y \rightarrow \infty$. En effet, notant classiquement

$$
\pi(y):=\sum_{p \leqslant y} 1, \quad \vartheta(y):=\sum_{p \leqslant y} \log p \asymp y \quad(y \geqslant 2),
$$

et posant traditionnellement $u:=(\log x) / \log y$, nous observons, à fins de référence ultérieure, que la définition (1.1) implique immédiatement

$$
\left(1+\frac{\vartheta(y)}{\log x}\right)^{u} \leqslant x^{\alpha} \leqslant \mathrm{e}^{\pi(y)} \quad(x \geqslant y \geqslant 2) .
$$

Pour améliorer la précision des résultats, nous introduisons les variables aléatoires géométriques indépendantes non biaisées $\xi_{p}^{*}=\xi_{p}^{*}(f)$ vérifiant

$$
\mathbb{P}\left(\xi_{p}^{*}=0\right)=g_{p}(\alpha), \quad \mathbb{P}\left(\xi_{p}^{*}=f\left(p^{\nu}\right)\right)=\frac{g_{p}(\alpha)}{\left(1-w_{p}\right) p^{\nu \alpha}} \quad\left(1 \leqslant \nu \leqslant \nu_{p}\right),
$$

avec la même convention que précédemment pour les cas d'égalité des valeurs prises, et nous posons

$$
Z_{f}^{*}:=\sum_{p \leqslant y} \xi_{p}^{*}
$$

Notons que, lorsque $f$ est fortement additive, les $\xi_{p}^{*}$ sont des variables de Bernoulli définies par

$$
\mathbb{P}\left(\xi_{p}^{*}=0\right)=g_{p}(\alpha), \quad \mathbb{P}\left(\xi_{p}^{*}=f(p)\right)=\frac{1}{p^{\alpha}} .
$$

Dans le cas général, nous avons

$$
\begin{aligned}
\mathbb{E}\left(Z_{f}^{*}\right) & =\sum_{p^{\nu} \in S(x, y)} \frac{g_{p}(\alpha) f\left(p^{\nu}\right)}{\left(1-w_{p}\right) p^{\nu \alpha}}, \\
\mathbb{V}\left(Z_{f}^{*}\right) & :=\sum_{p^{\nu} \in S(x, y)} \frac{g_{p}(\alpha)\left|f\left(p^{\nu}\right)\right|^{2}}{\left(1-w_{p}\right) p^{\nu \alpha}}-\sum_{p \leqslant y}\left|\sum_{1 \leqslant \nu \leqslant \nu_{p}} \frac{g_{p}(\alpha) f\left(p^{\nu}\right)}{\left(1-w_{p}\right) p^{\nu \alpha}}\right|^{2},
\end{aligned}
$$


la variance semi-empirique associée à ce modèle étant définie par

$$
V_{f}^{*}=V_{f}^{*}(x, y):=\frac{1}{\Psi(x, y)} \sum_{n \in S(x, y)}\left|f(n)-\mathbb{E}\left(Z_{f}^{*}\right)\right|^{2} .
$$

Notre approche fonctionne sans changement dans ce cadre : parallèlement au Théorème 1.1, nous obtenons le résultat suivant.

Théorème 1.2. Nous avons

$$
V_{f}^{*}(x, y) \ll \mathbb{V}\left(Z_{f}^{*}\right)
$$

uniformément pour toute fonction additive complexe $f$ et tous $x, y$ tels que $x \geqslant y \geqslant 2$.

Il est à noter que, lorsque $f$ est fortement additive, la variance de $Z_{f}^{*}$ prend la forme particulièrement agréable

$$
\mathbb{V}\left(Z_{f}^{*}\right)=\sum_{p \leqslant y} \frac{g_{p}(\alpha)|f(p)|^{2}}{p^{\alpha}}
$$

\section{Applications}

Nous présentons ici quelques exemples d'applications ouvertes par les Théorèmes 1.1 et 1.2 .

On sait classiquement que la forme duale de l'inégalité de Turán-Kubilius s'apparente à une inégalité de grand crible. Nous avons énoncé un tel résultat dans [3], comme conséquence de la majoration universelle (1·10). Comme le principe de dualité nécessite, au moins dans l'approche standard, une majoration par une forme quadratique diagonale, le recours à (1.6) ou (1.19) au lieu de (1.10) n'apporte pas d'amélioration immédiate. Cependant, dans le cas des fonctions fortement additives, la variance du modèle se présente sous forme diagonale. Nous obtenons le résultat suivant.

Théorème 2.1. Il existe une constante absolue $C>0$ telle que, pour toute suite $\left\{a_{n}\right\}_{n=1}^{\infty}$ de nombres complexes, et uniformément pour $x \geqslant y \geqslant 2$, on ait

$$
\sum_{p \leqslant y} \frac{p^{\alpha}}{g_{p}(\alpha)}\left|\sum_{\substack{n \in S(x, y) \\ p \mid n}} a_{n}-\frac{1}{p^{\alpha}} \sum_{n \in S(x, y)} a_{n}\right|^{2} \leqslant C \Psi(x, y) \sum_{n \in S(x, y)}\left|a_{n}\right|^{2} .
$$

Cela représente une amélioration significative par rapport au résultat analogue issu de $(1 \cdot 10)$, dans lequel les dénominateurs $g_{p}(\alpha)$ sont absents : $\operatorname{lorsque} y \leqslant \log x$, on a $g_{p}(\alpha) \asymp \pi(y)(\log p) / \log x \ll y / \log x$ et le gain est patent.

Il est également à noter que l'utilisation du modèle non biaisé $Z_{f}^{*}$ apporte aussi une précision supplémentaire : avec le modèle biaisé $Z_{f}$, nous n'aurions obtenu qu'une inégalité de type $(2 \cdot 1)$ où les dénominateurs $g_{p}(\alpha)$ sont remplacés par $g_{p}(\alpha)+w_{p}$.

Notre seconde application concerne la structure multiplicative d'un entier friable. Désignons par $\left\{p_{j}(n)\right\}_{j=1}^{\omega(n)}$ la suite croissante des facteurs premiers distincts d'un entier générique $n$, qui coïncide donc avec l'ensemble des sauts de la fonction

$$
t \mapsto \omega_{t}(n):=\sum_{\substack{p \mid n \\ p \leqslant t}} 1 \quad(2 \leqslant t \leqslant y)
$$


Plus précisément, nous avons $\omega_{t}(n)=j$ si, et seulement si, $p_{j}(n) \leqslant t<p_{j+1}(n)$, avec la convention que $p_{j+1}(n)=\infty$ lorsque $j=\omega(n)$. Comme $\omega_{t}(n)$ dépend additivement de $n$ pour chaque valeur de $t$ fixée, il est naturel d'attendre qu'une majoration de variance fournisse, via un argument de type Bienaymé-Tchébychev, une évaluation de $p_{j}(n)$ pour une sous-suite dense de $S(x, y)$. Nous avons développé une telle application dans [3] comme conséquence de (1·10). L'inégalité (1·19) permet de préciser le théorème 1.6 de [3].

Posons, pour $\alpha=\alpha(x, y)$,

$$
v_{t}:=\frac{t^{1-\alpha}-1}{(1-\alpha) \log t}, \quad M_{t}=M_{t}(x, y):=\sum_{p \leqslant t} \frac{1}{p^{\alpha}} \quad(2 \leqslant t \leqslant y \leqslant x) .
$$

Nous avons établi dans [3] que

$$
M_{t}=\log _{2} 2 t+v_{t}+O\left(\frac{v_{t}}{\log 2 v_{t}}\right) \quad(2 \leqslant t \leqslant y \leqslant x) .
$$

Nous obtenons le résultat suivant.

Théorème 2.2. Pour toute constante $b>1$ et toute fonction positive $\vartheta_{x}$ tendant vers l'infini avec $x$, il existe une fonction $\varepsilon_{x}$ telle que $\lim _{x \rightarrow \infty} \varepsilon_{x}=0$ ayant la propriété suivante : pour tous les entiers $n$ de $S(x, y)$ sauf au plus $\varepsilon_{x} \Psi(x, y)$ d'entre eux et uniformément pour $\vartheta_{x} \leqslant t \leqslant y \leqslant x$, nous avons

$$
\left|\omega_{t}(n)-M_{t}\right| \leqslant \begin{cases}\alpha^{1 / 3} M_{t}^{2 / 3}\left\{\log \left(3 M_{t} / \alpha\right)\right\}^{2 b / 3}+1 & (2 \leqslant t \leqslant z), \\ M_{t}^{2 / 3}\left(\log M_{t}\right)^{b / 3} & (z<t \leqslant y),\end{cases}
$$

où l'on a posé $z:=\min \{y, \exp (1 / \alpha)\}$.

Si, de plus, $\alpha=o(1)$, on peut choisir $\varepsilon_{x}$ de sorte que $\omega_{t}(n)=\pi(t)$ dès que $t=o(1 / \alpha)$.

Remarque. La formule $(2 \cdot 3)$ est essentiellement équivalente à

$$
\left|\omega_{t}(n)-M_{t}\right| \leqslant \frac{2 \alpha^{1 / 3} M_{t}^{2 / 3}\left\{\log \left(3 M_{t} / \alpha\right)\right\}^{2 b / 3}}{1+\left\{\alpha \log \left(3 M_{t} / \alpha\right)\right\}^{1 / 3}}+1 \quad(2 \leqslant t \leqslant y) .
$$

Le Théorème 2.2 permet une estimation de la répartition normale de la suite $\left\{p_{j}(n)\right\}_{j=1}^{\omega(n)}$. Qualitativement, lorsque, par exemple, $y \leqslant(\log x)^{k}$, où $k>1$ est une constante arbitraire fixée, nous mettons en évidence un remarquable effet de tassement, au point que $p_{j}(n)$ devient graduellement comparable, lorsque $y$ décroît, au $j$-ème nombre premier, noté $p_{j}$. Pour les plus grandes valeurs de $y$, nous avons $\alpha \gg 1$, et seule la seconde éventualité de $(2 \cdot 3)$ se présente. La conclusion du corollaire 1.7 de [3] fournit alors une description satisfaisante de la situation.

Nous posons $z_{1}:=\min \left(y, \mathrm{e}^{1 / 2 \alpha}\right)$ et notons que $\alpha \leqslant 1-1 / k+o(1)$ pour $y \leqslant(\log x)^{k}$.

Corollaire 2.3. Soit $k>1$. Pour toute constante $b>1$ et toute fonction positive $J_{x}$ tendant vers l'infini avec $x$, il existe une fonction $\varepsilon_{x}$ telle que $\lim _{x \rightarrow \infty} \varepsilon_{x}=0$ et vérifiant la propriété suivante : pour tous les entiers n de $S(x, y)$ sauf au plus $\varepsilon_{x} \Psi(x, y)$ d'entre eux et pour $J_{x} \leqslant j \leqslant \omega(n), 2 \leqslant y \leqslant(\log x)^{k}$, nous avons

$$
\begin{gathered}
0 \leqslant p_{j}(n)-p_{j} \ll \alpha^{1 / 3} p_{j}^{2 / 3}\left\{\log \left(3 p_{j} / \alpha\right)\right\}^{2 b / 3}+\alpha p_{j}+1 \quad\left(p_{j} \leqslant z_{1}\right) \\
p_{j}(n)=p_{j}^{1 /(1-\alpha)} \mathrm{e}^{O(\alpha)} \quad\left(z_{1}<p_{j} \leqslant y\right) .
\end{gathered}
$$

De plus, lorsque $\alpha=o(1)$, on a $p_{j}(n)=p_{j}$ dès que $p_{j}=o(1 / \alpha)$.

Il est à noter que l'énoncé précédent implique en particulier que presque tous les entiers de $S(x, y)$ vérifient

$$
p_{j}(n) \sim p_{j} \quad(1 \leqslant j \leqslant \omega(n))
$$

dès que $y=o(\log x)$. 


\section{Démonstration du Théorème 1.1}

Soit $f \in \mathbb{A}$. Étant donnés $x, y$ tels que $x \geqslant y \geqslant 2$, nous définissons une fonction fortement additive $h=h_{x, y}$ par

$$
\frac{h(p)}{p^{\alpha}}\left(1-w_{p}\right)=\sum_{1 \leqslant \nu \leqslant \nu_{p}} g_{p}(\alpha) \frac{f\left(p^{\nu}\right)}{p^{\nu \alpha}} \quad(p \in \mathcal{P}),
$$

où nous avons utilisé les notations introduites en (1·11).

Nous avons donc $f=g+h$, où $g$ vérifie (1.7). Pour un couplage convenable, nous avons également $Z_{f}=Z_{g}+Z_{h}$ : il suffit en effet de choisir, avec la convention indiquée dans l'introduction,

$$
\left\{\begin{aligned}
& \mathbb{P}\left(\xi_{p}(h)=0, \xi_{p}(g)=0\right):=g_{p}(\alpha)+\frac{w_{p}}{p^{\alpha}}, \\
& \mathbb{P}\left(\xi_{p}(h)=h(p), \xi_{p}(g)=g\left(p^{\nu}\right)\right):=\frac{g_{p}(\alpha)}{p^{\nu \alpha}} \quad\left(1 \leqslant \nu \leqslant \nu_{p}\right) .
\end{aligned}\right.
$$

Ainsi qu'il a été rappelé plus haut, il résulte du théorème 1.1 de [3] que

$$
V_{g}=V_{g}(x, y) \ll \mathbb{V}\left(Z_{g}\right) .
$$

De plus,

$$
\begin{aligned}
V_{f} & \leqslant 2 V_{g}+2 V_{h}, \\
\mathbb{V}\left(Z_{h}\right) & =\sum_{p \leqslant y}\left(1-w_{p}\right) \frac{|h(p)|^{2}}{p^{\alpha}}\left(1-\frac{1-w_{p}}{p^{\alpha}}\right)=\sum_{p \leqslant y}\left(1-w_{p}\right) \frac{|h(p)|^{2}}{p^{\alpha}}\left(g_{p}(\alpha)+\frac{w_{p}}{p^{\alpha}}\right) .
\end{aligned}
$$

Observons alors, d'une part, que, pour tout nombre premier $p \leqslant y$,

$$
\begin{aligned}
\left(1-w_{p}\right) \frac{|h(p)|^{2}}{p^{\alpha}} & \leqslant \frac{p^{\alpha}}{1-w_{p}} \sum_{1 \leqslant \nu \leqslant \nu_{p}} \frac{g_{p}(\alpha)}{p^{\nu \alpha}} \sum_{1 \leqslant \nu \leqslant \nu_{p}} \frac{g_{p}(\alpha)}{p^{\nu \alpha}}\left|f\left(p^{\nu}\right)\right|^{2} \\
& =\sum_{1 \leqslant \nu \leqslant \nu_{p}} \frac{g_{p}(\alpha)}{p^{\nu \alpha}}\left|f\left(p^{\nu}\right)\right|^{2} .
\end{aligned}
$$

alors que, d'autre part, par un emploi standard de l'inégalité de Cauchy-Schwarz,

$$
\begin{aligned}
\mathbb{V}\left(Z_{f}\right) & =\sum_{p^{\nu} \in S(x, y)} \frac{g_{p}(\alpha)}{p^{\nu \alpha}}\left|f\left(p^{\nu}\right)\right|^{2}-\sum_{p \leqslant y}\left|\sum_{1 \leqslant \nu \leqslant \nu_{p}} \frac{g_{p}(\alpha)}{p^{\nu \alpha}} f\left(p^{\nu}\right)\right|^{2} \\
& \geqslant \sum_{p^{\nu} \in S(x, y)} \frac{g_{p}(\alpha)}{p^{\nu \alpha}}\left|f\left(p^{\nu}\right)\right|^{2}\left(g_{p}(\alpha)+\frac{w_{p}}{p^{\alpha}}\right) .
\end{aligned}
$$

Il s'ensuit que donc

$$
\mathbb{V}\left(Z_{h}\right) \leqslant \mathbb{V}\left(Z_{f}\right) .
$$

Sous l'hypothèse $V_{h} \ll \mathbb{V}\left(Z_{h}\right)$, nous déduisons donc de ce qui précède que nous avons

$$
V_{f} \leqslant 2 V_{g}+2 V_{h} \ll \mathbb{V}\left(Z_{f}-Z_{h}\right)+\mathbb{V}\left(Z_{h}\right) \ll \mathbb{V}\left(Z_{f}\right)+\mathbb{V}\left(Z_{h}\right) \ll \mathbb{V}\left(Z_{f}\right),
$$

c'est-à-dire (1·6).

Nous pouvons ainsi, sans restreindre la généralité de l'argument, supposer dans la suite que $f$ est une fonction fortement additive. 
Nous pouvons également supposer $f$ réelle puisque

$$
V_{f}=V_{\Re e f}+V_{\Im m f}, \quad \mathbb{V}\left(Z_{f}\right)=\mathbb{V}\left(Z_{\Re e f}\right)+\mathbb{V}\left(Z_{\Im m f}\right)
$$

dans le cas général.

À fins de référence ultérieure, nous notons immédiatement que

$$
\mathbb{V}\left(Z_{f}\right)=\sum_{p \leqslant y}\left(1-w_{p}\right) \frac{f(p)^{2}}{p^{\alpha}}\left(g_{p}(\alpha)+\frac{w_{p}}{p^{\alpha}}\right)
$$

et rappelons la formule $(1 \cdot 20)$ pour $\mathbb{V}\left(Z_{f}^{*}\right)$.

Sous les hypothèses supplémentaires mentionnées plus haut, nous pouvons écrire

$$
V_{f}(x, y)=\frac{1}{\Psi(x, y)} \sum_{n \in S(x, y)}\left(\sum_{p \mid n} f(p)-\mathbb{E}\left(Z_{f}\right)\right)^{2}=M_{2}-2 M_{1} \mathbb{E}\left(Z_{f}\right)+\mathbb{E}\left(Z_{f}\right)^{2}
$$

avec

$$
\mathbb{E}\left(Z_{f}\right)=\sum_{p \leqslant y} \frac{f(p)\left(1-w_{p}\right)}{p^{\alpha}}, \quad M_{j}:=\frac{1}{\Psi(x, y)} \sum_{n \in S(x, y)} f(n)^{j} \quad(j=1,2),
$$

de sorte que

$$
M_{1}=\sum_{p \leqslant y} f(p) \frac{\Psi(x / p, y)}{\Psi(x, y)}, \quad M_{2}=\sum_{p \leqslant y} f(p)^{2} \frac{\Psi(x / p, y)}{\Psi(x, y)}+\sum_{\substack{p, q \leqslant y \\ p \neq q}} f(p) f(q) \frac{\Psi(x / p q, y)}{\Psi(x, y)} .
$$

Il est manifeste que des estimations relatives au comportement local de $\Psi(x, y)$ sont nécessaires pour évaluer $M_{1}$ et $M_{2}$. Nous ferons appel à la formule générale suivante, établie au théorème 2.4 de [2] et valable uniformément sous les conditions $x \geqslant y \geqslant 2$, $1 \leqslant d \leqslant x, \log d \ll \log y, \omega(m) \ll 1$ :

$$
\Psi_{m}\left(\frac{x}{d}, y\right):=\sum_{\substack{n \in S(x / d, y) \\(n, m)=1}} 1=\frac{g_{m}(\alpha)}{d^{\alpha}} \Psi(x, y)\left\{1+O\left(\frac{1}{\bar{u}}\right)\right\}
$$

où l'on a posé

$$
\bar{u}:=\min (u, y / \log y) .
$$

Introduisant

$$
r_{d}=r_{d}(x, y):=\frac{\Psi(x / d, y)}{\Psi(x, y)}-\frac{1}{d^{\alpha}} \quad(x \geqslant y \geqslant 2, d \geqslant 1)
$$

nous obtenons en appliquant $(3 \cdot 4)$ avec $m=1, d=p$, puis $m=p, d=1$,

$$
r_{p}=\frac{\Psi(x / p, y)}{\Psi(x, y)}-\frac{1}{p^{\alpha}}=g_{p}(\alpha)-\frac{\Psi_{p}(x, y)}{\Psi(x, y)} \ll \frac{g_{p}(\alpha)}{p^{\alpha} \bar{u}},
$$

uniformément pour $x \geqslant y \geqslant 2, p \leqslant y$. 
Compte tenu des résultats rappelés dans l'introduction, nous pouvons supposer

$$
2 \leqslant y \leqslant \log x
$$

de sorte que $y^{\alpha} \asymp 1$ - cf., par exemple, [7] ou (5.15) infra. Observons d'emblée qu'à toute fin utile nous pouvons remplacer, dans le membre de droite de $(3 \cdot 3)$, l'espérance du modèle $Z_{f}$ par celle du modèle non biaisé $Z_{f}^{*}$, défini en $(1 \cdot 15)$. En effet, puisque

$$
\mathbb{V}\left(Z_{f}\right)=\sum_{p \leqslant y}\left(1-w_{p}\right) \frac{f(p)^{2}}{p^{\alpha}}\left(g_{p}(\alpha)+\frac{w_{p}}{p^{\alpha}}\right) \geqslant\left\{1-(y / x)^{\alpha}\right\} \sum_{p \leqslant y} \frac{f(p)^{2} w_{p}}{p^{\alpha}},
$$

nous avons, d'une part,

$$
\left\{\mathbb{E}\left(Z_{f}\right)-\mathbb{E}\left(Z_{f}^{*}\right)\right\}^{2} \leqslant \sum_{p \leqslant y} \frac{f(p)^{2} w_{p}}{p^{\alpha}} \sum_{p \leqslant y} \frac{w_{p}}{p^{\alpha}} \ll \frac{\pi(y) x^{-\alpha}}{1-(y / x)^{\alpha}} \mathbb{V}\left(Z_{f}\right) \ll \frac{y \mathbb{V}\left(Z_{f}\right)}{x^{\alpha} \log y}
$$

et, d'autre part, au vu de (3.6), puisque $\alpha \leqslant \pi(y) / \log x$ d'après (1.13) et donc $p^{\alpha} \ll 1$ pour $p \leqslant y$ dans le domaine $(3 \cdot 7)$,

$$
\begin{aligned}
& M_{1}-\mathbb{E}\left(Z_{f}^{*}\right)=\sum_{p \leqslant y} f(p) r_{p} \ll \frac{1}{\pi(y)} \sqrt{\sum_{p \leqslant y} \frac{f(p)^{2} g_{p}(\alpha)}{p^{\alpha}} \sum_{p \leqslant y} \frac{g_{p}(\alpha)}{p^{\alpha}}} \ll \sqrt{\frac{\mathbb{V}\left(Z_{f}^{*}\right)}{u}} \\
& M_{1}-\mathbb{E}\left(Z_{f}\right)=\sum_{p \leqslant y} f(p)\left\{r_{p}+\frac{w_{p}}{p^{\alpha}}\right\} \ll \sqrt{\frac{\mathbb{V}\left(Z_{f}^{*}\right)}{u}}+\sqrt{\frac{\pi(y) \mathbb{V}\left(Z_{f}\right)}{x^{\alpha}}}
\end{aligned}
$$

Puisque $V_{f}^{*}=V_{f}^{*}(x, y)=M_{2}-2 M_{1} \mathbb{E}\left(Z_{f}^{*}\right)+\mathbb{E}\left(Z_{f}^{*}\right)^{2}$, il s'ensuit que

$$
\begin{aligned}
V_{f}-V_{f}^{*} & =\left\{\mathbb{E}\left(Z_{f}^{*}\right)-\mathbb{E}\left(Z_{f}\right)\right\}\left\{2 M_{1}-\mathbb{E}\left(Z_{f}\right)-\mathbb{E}\left(Z_{f}^{*}\right)\right\} \\
& \ll\left\{\sqrt{\frac{y}{x^{\alpha} \log x}}+\frac{y}{x^{\alpha} \log y}\right\} \mathbb{V}\left(Z_{f}\right) \ll \mathbb{V}\left(Z_{f}\right) .
\end{aligned}
$$

Estimons donc $V_{f}^{*}$. Nous avons

$$
\begin{aligned}
M_{2} & =\sum_{p \leqslant y} \frac{f(p)^{2}}{p^{\alpha}}+\sum_{p \leqslant y} f(p)^{2} r_{p}+\sum_{\substack{p, q \leqslant y \\
p \neq q}} \frac{f(p) f(q)}{p^{\alpha} q^{\alpha}}+\sum_{\substack{p, q \leqslant y \\
p \neq q}} f(p) f(q) r_{p q} \\
& =\mathbb{V}\left(Z_{f}^{*}\right)+\mathbb{E}\left(Z_{f}^{*}\right)^{2}+\sum_{p \leqslant y} f(p)^{2} r_{p}+\sum_{\substack{p, q \leqslant y \\
p \neq q}} f(p) f(q) r_{p q},
\end{aligned}
$$

d'où, compte tenu de l'égalité de (3·10),

$$
V_{f}^{*}=\mathbb{V}\left(Z_{f}^{*}\right)-G_{f}+D_{f}
$$

avec

$$
\begin{gathered}
\mathbb{V}\left(Z_{f}^{*}\right)=\sum_{p \leqslant y} \frac{g_{p}(\alpha) f(p)^{2}}{p^{\alpha}} \leqslant \frac{\mathbb{V}\left(Z_{f}\right)}{1-(y / x)^{\alpha}}=\left\{1+O\left(\frac{1}{x^{\alpha}}\right)\right\} \mathbb{V}\left(Z_{f}\right), \\
G_{f}:=\sum_{p \leqslant y} f(p)^{2} r_{p}\left(\frac{2}{p^{\alpha}}-1\right), \quad D_{f}:=\sum_{\substack{p, q \leqslant y \\
p \neq q}} f(p) f(q)\left\{r_{p q}-\frac{r_{p}}{q^{\alpha}}-\frac{r_{q}}{p^{\alpha}}\right\},
\end{gathered}
$$

où $(3 \cdot 14)$ découle de $(3 \cdot 8)$. 
Il résulte immédiatement de $(3 \cdot 6)$ que

$$
G_{f} \ll \frac{\mathbb{V}\left(Z_{f}^{*}\right)}{\pi(y)}
$$

Nous avons également

$$
r_{p q}-\frac{r_{p}}{q^{\alpha}}-\frac{r_{q}}{p^{\alpha}}=\frac{1}{\Psi(x, y)}\left\{\Psi\left(\frac{x}{p q}, y\right)-\frac{\Psi(x / p, y)}{q^{\alpha}}-\frac{\Psi(x / q, y)}{p^{\alpha}}+\frac{\Psi(x, y)}{p^{\alpha} q^{\alpha}}\right\} .
$$

La quantité entre accolades vaut

$$
\begin{aligned}
\sum_{t \mid p q} \mu(t)\left(\frac{t}{p q}\right)^{\alpha} \Psi\left(\frac{x}{t}, y\right) & =\sum_{t \mid p q} \mu(t) \sum_{d \mid p q / t} g_{d}(\alpha) \mu(d) \sum_{\substack{n \in S(x, y) \\
t \mid n}} 1 \\
& =\sum_{d \mid p q} \mu(d) g_{d}(\alpha) \sum_{n \in S(x, y)} \sum_{t \mid(n, p q / d)} \mu(t) \\
& =\sum_{d \mid p q} \mu(d) g_{d}(\alpha) \Psi_{p q / d}(x, y) .
\end{aligned}
$$

Compte tenu de $(3 \cdot 4)$, le membre de droite de $(3 \cdot 18)$ peut être évalué par

$$
\sum_{d \mid p q} \frac{\mu(d) d^{\alpha} g_{d}(\alpha) g_{p q / d}(\alpha)}{d^{\alpha} p^{\alpha} q^{\alpha}} \Psi(x, y)+O\left(\frac{g_{p q}(\alpha)}{\pi(y) p^{\alpha} q^{\alpha}} \Psi(x, y)\right) \ll \frac{g_{p q}(\alpha)}{\pi(y) p^{\alpha} q^{\alpha}} \Psi(x, y) .
$$

Nous obtenons donc

$$
D_{f} \ll \frac{1}{\pi(y)}\left(\sum_{p \leqslant y} g_{p}(\alpha) \frac{|f(p)|}{p^{\alpha}}\right)^{2} \leqslant \frac{\mathbb{V}\left(Z_{f}^{*}\right)}{\pi(y)} \sum_{p \leqslant y} \frac{g_{p}(\alpha)}{p^{\alpha}} .
$$

La dernière somme est $\ll \alpha y \ll y \pi(y) / \log x$. Il suit

$$
D_{f} \ll \frac{y}{\log x} \mathbb{V}\left(Z_{f}^{*}\right)
$$

La relation $(3 \cdot 13)$ et les estimations $(3 \cdot 12),(3 \cdot 14),(3 \cdot 16)$ et $(3 \cdot 19)$ fournissent alors

$$
V_{f}(x, y)=\mathbb{V}\left(Z_{f}\right)\left\{1+O\left(\frac{\log y}{y}+\frac{y}{\log x}\right)\right\} \quad(2 \leqslant y \leqslant \log x),
$$

ce qui complète la preuve du Théorème 1.1.

À fins de référence ultérieure, nous observons que la démonstration précédente fournit également

$$
V_{f}^{*}(x, y)=\mathbb{V}\left(Z_{f}^{*}\right)\left\{1+O\left(\frac{\log y}{y}+\frac{y}{\log x}\right)\right\} \quad(2 \leqslant y \leqslant \log x) .
$$




\section{Démonstration du Théorème 1.2}

Comme précédemment, il suffit de considérer le cas des fonctions fortement additives. En effet, étant donnés une fonction réelle $f \in \mathbb{A}$ et $x, y$ tels que $x \geqslant y \geqslant 2$, la fonction $g:=f-h$, où $h=h_{x, y}$ est définie par $(3 \cdot 1)$, vérifie $\mathbb{E}\left(\xi_{p}(g)\right)=\mathbb{E}\left(\xi_{p}^{*}(g)\right)=0$ pour tout $p \leqslant y$. Nous pouvons donc appliquer le théorème 1.1 de [3], qui fournit

$$
V_{g}(x, y) \ll \mathbb{V}\left(Z_{g}\right) \asymp \mathbb{V}\left(Z_{g}^{*}\right)
$$

puisque $1-w_{p} \asymp 1$ pour $p \leqslant y$. De plus, pour un couplage convenable, nous avons la relation entre variables aléatoires $Z_{f}^{*}=Z_{g}^{*}+Z_{h}^{*}$, de sorte que $\mathbb{V}\left(Z_{g}^{*}\right) \leqslant 2 \mathbb{V}\left(Z_{h}^{*}\right)+2 \mathbb{V}\left(Z_{f}^{*}\right)$.

Maintenant

$$
\begin{aligned}
V_{g}^{*}-2 V_{g} \leqslant 2\left|\mathbb{E}\left(Z_{g}\right)-\mathbb{E}\left(Z_{g}^{*}\right)\right|^{2} & \ll \sum_{p \leqslant y} \sum_{1 \leqslant \nu \leqslant \nu_{p}} \frac{g_{p}(\alpha)\left|g\left(p^{\nu}\right)\right|^{2}}{p^{\nu \alpha}} \sum_{p \leqslant y} w_{p}^{2} \sum_{1 \leqslant \nu \leqslant \nu_{p}} \frac{g_{p}(\alpha)}{p^{\nu \alpha}} \\
& \ll \mathbb{V}\left(Z_{g}^{*}\right) \frac{\pi(y)}{x^{2 \alpha}} \ll \mathbb{V}\left(Z_{g}^{*}\right),
\end{aligned}
$$

d'où

$$
V_{g}^{*} \ll \mathbb{V}\left(Z_{g}^{*}\right) \ll \mathbb{V}\left(Z_{h}^{*}\right)+\mathbb{V}\left(Z_{f}^{*}\right)
$$

De plus,

$$
\begin{aligned}
\mathbb{V}\left(Z_{h}^{*}\right) & =\sum_{p \leqslant y} \frac{g_{p}(\alpha)|h(p)|^{2}}{p^{\alpha}} \leqslant \sum_{p \leqslant y} \frac{g_{p}(\alpha)}{\left(1-w_{p}\right)^{2}} \sum_{1 \leqslant \nu \leqslant \nu_{p}} \frac{g_{p}(\alpha)\left|f\left(p^{\nu}\right)\right|^{2}}{p^{\nu \alpha}} \sum_{1 \leqslant \nu \leqslant \nu_{p}} \frac{g_{p}(\alpha)}{p^{(\nu-1) \alpha}} \\
& \leqslant \sum_{p^{\nu} \in S(x, y)} \frac{g_{p}(\alpha)^{2}\left|f\left(p^{\nu}\right)\right|^{2}}{\left(1-w_{p}\right) p^{\alpha}} \ll \mathbb{V}\left(Z_{f}^{*}\right) .
\end{aligned}
$$

Sous l'hypothèse $V_{h}^{*} \ll V\left(Z_{h}^{*}\right)$, il suit donc

$$
V_{f}^{*} \leqslant 2 V_{g}^{*}+2 V_{h}^{*} \ll \mathbb{V}\left(Z_{g}^{*}\right)+\mathbb{V}\left(Z_{h}^{*}\right) \ll \mathbb{V}\left(Z_{f}^{*}\right)+\mathbb{V}\left(Z_{h}^{*}\right) \ll \mathbb{V}\left(Z_{f}^{*}\right) .
$$

Il reste à établir que $V_{h}^{*} \ll V\left(Z_{h}^{*}\right)$. Cela résulte de (3.21) lorsque $2 \leqslant y \leqslant \log x$. Lorsque $\log x<y \leqslant x$, nous avons $w_{p} / p^{\alpha} \ll g_{p}(\alpha)$ pour tout $p \leqslant y$, donc $\mathbb{V}\left(Z_{h}\right) \asymp \mathbb{V}\left(Z_{h}^{*}\right)$, et, grâce au théorème 1.1 de [3]

$$
\begin{aligned}
V_{h}^{*} & \leqslant 2 V_{h}+2\left\{\mathbb{E}\left(Z_{h}\right)-\mathbb{E}\left(Z_{h}^{*}\right)\right\}^{2} \ll \mathbb{V}\left(Z_{h}\right)+\sum_{p \leqslant y} \frac{w_{p}^{2}}{p^{\alpha}} \sum_{p \leqslant y} \frac{|f(p)|^{2}}{p^{\alpha}} \\
& \ll \mathbb{V}\left(Z_{h}\right)+\frac{\pi(y)}{x^{3 \alpha / 2}} B_{h}(x, y)^{2} \ll \mathbb{V}\left(Z_{h}\right) \ll \mathbb{V}\left(Z_{h}^{*}\right),
\end{aligned}
$$

où l'avant-dernière estimation résulte de (1.9) et (1·13).

\section{Le cas des fonctions fortement additives}

\section{1. Énoncé du résultat principal et remarques}

La démonstration du Théorème 1.1 fait apparaître le rôle essentiel joué par la classe des fonctions fortement additives dans l'étude du cas général. Nous rassemblons nos résultats relatifs à cette hypothèse dans l'énoncé suivant.

Nous posons

$$
\begin{aligned}
\eta=\eta(x, y) & :=\frac{1}{u}+\frac{y^{2} \pi(y)}{(\log x)^{2}}+\frac{y^{3} \pi(y)^{2}}{(\log x)^{3}} \\
\mathcal{R}=\mathcal{R}(x, y) & :=\min \left\{\frac{\log y}{y}+\frac{y}{\log x}, \eta(x, y)\right\}
\end{aligned}
$$


et notons que $\eta=o(1)$ si, et seulement si, $y=o\left((\log x)^{3 / 5}\left(\log _{2} x\right)^{2 / 5}\right)$. Nous rappelons également la notation de Tchébychev

$$
\vartheta(y):=\sum_{p \leqslant y} \log p
$$

Par ailleurs, nous désignons par $\mathbb{A}_{0}$ la classe des fonctions arithmétiques réelles fortement additives.

Théorème 5.1. Soit $f \in \mathbb{A}_{0}$.

(i) Nous avons

$$
V_{f}^{*}(x, y) \leqslant\{1+o(1)\} \mathbb{V}\left(Z_{f}^{*}\right) \quad(u \rightarrow \infty) .
$$

Si, en outre, $y=o(\log x)$, alors

$$
V_{f}^{*}(x, y)=\{1+o(1)\} \mathbb{V}\left(Z_{f}^{*}\right)
$$

(ii) Lorsque $y$ et $u$ tendent vers l'infini, nous avons également

$$
V_{f}(x, y) \leqslant\{1+o(1)\} \mathbb{V}\left(Z_{f}\right)
$$

(iii) Si $y$ et $(\log x) / y$ tendent vers l'infini, alors

$$
\frac{1}{1+(\log x) / \mathrm{e}^{\pi(y)} \pi(y) \log 2}+o(1) \leqslant \frac{V_{f}(x, y)}{\mathbb{V}\left(Z_{f}\right)} \leqslant \frac{1}{1+(\log x) / y \mathrm{e}^{\pi(y)}}+o(1) .
$$

(iv) Sous l'hypothèse $2 \leqslant y \leqslant \log x$, nous avons plus précisément

$$
1-\frac{\vartheta(y)}{\log x}+O(\mathcal{R}) \leqslant \frac{V_{f}^{*}(x, y)}{\mathbb{V}\left(Z_{f}^{*}\right)} \leqslant 1+O(\mathcal{R})
$$

Remarques. (i) La formule (5.3) met en évidence l'indépendance asymptotique, dans le domaine $y=o(\log x)$, des antécédents arithmétiques des variables $\xi_{p}^{*}$ associées aux fonctions fortement additives selon le modèle non biaisé.

(ii) Martin et Tenenbaum ont montré - [8], formule (13.6) — que, pour tout $u \geqslant 1$ fixé, nous avons

$$
\limsup _{x \rightarrow \infty} \sup _{f \in \mathbb{A}_{0}} \frac{V_{f}\left(x, x^{1 / u}\right)}{\mathbb{V}\left(Z_{f}\right)}=\lambda(u)
$$

pour une application convenable $\lambda:\left[1, \infty\left[\rightarrow \mathbb{R}^{+}\right.\right.$, précisément définie dans le même travail et vérifiant $\lambda(u)-1 \sim 1 / 8 u(u \rightarrow \infty)$. Les valeurs de $\lambda(u)$ ont été tabulées dans [6], et vérifient $\lambda(u)>1$ pour tout $u>1$. Cela implique en particulier l'optimalité du domaine de validité de $(5 \cdot 2)$. 
(iii) La formule (5.4) est une conséquence immédiate de $(5 \cdot 2),(3 \cdot 12)$ et $(3 \cdot 14)$. Dans le domaine $y \leqslant \log x$, on obtient en fait une estimation effective en ajoutant $\pi(y) \mathrm{e}^{-\pi(y)}$ aux termes d'erreur de $(5 \cdot 6)$.

(iv) Lorsque $y=2$, il est aisé d'estimer $V_{f}(x, y) / \mathbb{V}\left(Z_{f}\right)$. Pour des raisons d'homogénéité, nous pouvons supposer $f(2)=1$. Posant $N:=\Psi(x, 2)=1+\nu_{2}(x)$, nous avons $\alpha=(1+O(1 / N)) / N \log 2$ et

$$
\begin{aligned}
\mathbb{E}\left(Z_{f}\right) & =2^{-\alpha}\left(1-2^{-N \alpha}\right)=1-\mathrm{e}^{-1}+O(1 / N), \\
V_{f} & =\mathrm{e}^{-2}+O(1 / N), \\
\mathbb{V}\left(Z_{f}\right) & =\mathrm{e}^{-1}-\mathrm{e}^{-2}+O(1 / N),
\end{aligned}
$$

de sorte que

$$
\frac{V_{f}(x, y)}{\mathbb{V}\left(Z_{f}\right)}=\frac{1}{\mathrm{e}-1}+O\left(\frac{1}{N}\right)<1 .
$$

(v) Désignons, conformément à l'usage, par $\omega(n)$ le nombre des facteurs premiers distincts d'un entier naturel $n$. Comme nous l'établirons au paragraphe 5.4, nous avons lorsque $(\log x) / y$ et $y$ tendent vers l'infini,

$$
V_{\omega}=\left(\frac{1}{1+(\log x) / y \mathrm{e}^{\pi(y)}}+o(1)\right) \mathbb{V}\left(Z_{\omega}\right) .
$$

Cela implique l'optimalité de la majoration de (5.5) dans la généralité énoncée.

(vi) Le choix de la fonction indicatrice des nombres pairs permet de montrer que la minoration de $(5 \cdot 5)$ est également optimale dans la généralité énoncée : il suffit de faire appel à $(3 \cdot 2),(1 \cdot 20)$ et $(5 \cdot 15),(5 \cdot 27)$ infra. Nous omettons les détails de la vérification.

\subsection{Lemmes}

Commençons par affiner les estimations $(3 \cdot 16)$ et $(3 \cdot 19)$ dans le domaine $(3 \cdot 7)$. Rappelons la notation $(5 \cdot 1)$.

Lemme 5.2. Dans le domaine (3·7), uniformément pour $f \in \mathbb{A}_{0}$, nous avons

$$
G_{f} \ll \eta \mathbb{V}\left(Z_{f}^{*}\right)
$$

et, pour une constante absolue convenable $K$,

$$
-\mathbb{V}\left(Z_{f}^{*}\right)\left\{\frac{\vartheta(y)}{\log x}+K \eta\right\} \leqslant D_{f} \leqslant K \eta \mathbb{V}\left(Z_{f}^{*}\right) .
$$

Remarque. L'estimation (5.9) fournit une amélioration de (3.16) dès que

$$
y \ll \sqrt{\log x \log _{2} x} .
$$

Démonstration. Considérons d'abord (5·9). Posons

$$
\delta=\delta(x, y):=\frac{1}{u}+\frac{y^{2} \pi(y)}{(\log x)^{2}} .
$$

Dans le domaine $(3 \cdot 7)$ et pour $2 \leqslant p \leqslant y$, le corollaire 1.8 de [5] implique

$$
\Psi_{p}(x, y)=g_{p}(\alpha) \Psi(x, y)\{1+O(\delta)\},
$$

d'où

$$
r_{p} \ll g_{p}(\alpha) \delta,
$$

et finalement (5.9). 
Nous procédons similairement pour établir la majoration de $(5 \cdot 10)$. Une forme affaiblie du corollaire 1.8 de [5] permet d'écrire

$$
\Psi_{p q}(x, y)=g_{p q}(\alpha) \Psi(x, y)\left\{1-\frac{1}{\pi(y)}+O(\delta)\right\},
$$

d'où, compte tenu de $(3 \cdot 17)$ et $(3 \cdot 18)$,

$$
\begin{aligned}
r_{p q}-\frac{r_{p}}{q^{\alpha}}-\frac{r_{q}}{p^{\alpha}} & =\sum_{d \mid p q} \mu(d) g_{d}(\alpha) \frac{\Psi_{p q / d}(x, y)}{\Psi(x, y)} \\
& =g_{p q}(\alpha)\left\{-\frac{1}{\pi(y)}+O(\delta)\right\}=\frac{-g_{p q}(\alpha)}{p^{\alpha} q^{\alpha} \pi(y)}+O\left(\frac{g_{p q}(\alpha) \delta}{p^{\alpha} q^{\alpha}}\right),
\end{aligned}
$$

où nous avons utilisé l'estimation $p^{\alpha} q^{\alpha}=1+O(y / \log x)$, qui résulte de $(1 \cdot 13)$.

Reportons cette estimation dans l'expression de $D_{f}$ donnée en (3·15). Le terme d'erreur fournit une contribution $\ll \eta \mathbb{V}\left(Z_{f}^{*}\right)$. Il s'ensuit que, dans le domaine (3.7), nous avons

$$
\begin{aligned}
D_{f} & =-\frac{1}{\pi(y)}\left(\sum_{p \leqslant y} \frac{f(p) g_{p}(\alpha)}{p^{\alpha}}\right)^{2}+\frac{1}{\pi(y)} \sum_{p \leqslant y} \frac{f(p)^{2} g_{p}(\alpha)^{2}}{p^{2 \alpha}}+O\left(\alpha y \delta \mathbb{V}\left(Z_{f}^{*}\right)\right) \\
& \leqslant K \eta \mathbb{V}\left(Z_{f}^{*}\right),
\end{aligned}
$$

pour une constante absolue convenable $K$, puisque $g_{p}(\alpha) / \pi(y) \ll 1 / u \ll \eta$ dans le domaine considéré.

Pour établir la minoration de (5·10), nous appliquons l'inégalité de Cauchy-Schwarz sous la forme

$$
\begin{aligned}
\left(\sum_{p \leqslant y} \frac{f(p) g_{p}(\alpha)}{p^{\alpha}}\right)^{2} & \leqslant \mathbb{V}\left(Z_{f}^{*}\right) \sum_{p \leqslant y} \frac{g_{p}(\alpha)}{p^{\alpha}} \leqslant \mathbb{V}\left(Z_{f}^{*}\right) \alpha \sum_{p \leqslant y} \log p \\
& \leqslant \mathbb{V}\left(Z_{f}^{*}\right) \frac{\pi(y) \vartheta(y)}{\log x}\left\{1+O\left(\frac{y}{\log x}\right)\right\}
\end{aligned}
$$

où nous avons utilisé l'estimation (voir la formule (2.14) de [5])

$$
\alpha=\frac{\pi(y)}{\log x}\left\{1+O\left(\frac{y}{\log x}\right)\right\} \quad(2 \leqslant y \leqslant \log x) .
$$

Nous en déduisons l'inégalité souhaitée en reportant dans l'égalité de $(5 \cdot 14)$.

Lemme 5.3. Soit $h:\left[1, \infty\left[\rightarrow \mathbb{R}^{+}\right.\right.$une fonction telle que $\lim _{x \rightarrow \infty} h(x)=\infty$. Sous la condition

$$
h(x) \sqrt{\log x} \log _{2} x \leqslant y \leqslant x^{1 / h(x)},
$$

et uniformément pour $f \in \mathbb{A}_{0}$, nous avons

$$
V_{f}(x, y) \leqslant\{1+o(1)\} \mathbb{V}\left(Z_{f}\right) \quad(x \rightarrow \infty) .
$$

Démonstration. Lorsque $(5 \cdot 16)$ est réalisée et, sous la condition supplémentaire $u>\sqrt{\log y}$, la preuve du théorème principal de [4] fournit en fait l'existence d'une constante $K>0$ 
telle que pour toute fonction $f$ additive réelle

$$
V_{f}(x, y)-\mathbb{V}\left(Z_{f}\right) \leqslant K \frac{B^{2}(x, y)}{\bar{u}} \cdot(2)
$$

Lorsque $f$ est fortement additive, l'inégalité $\frac{1}{2} \alpha \log 2 \leqslant g_{p}(\alpha)$ permet d'en déduire que

$$
V_{f}(x, y)-\mathbb{V}\left(Z_{f}\right) \leqslant \frac{K}{\bar{u}} \sum_{p \leqslant y} \frac{f(p)^{2}}{p^{\alpha}} \leqslant \frac{2 K}{\bar{u} \alpha \log 2} \mathbb{V}\left(Z_{f}^{*}\right) .
$$

La minoration classique $\alpha \log y \gg \log (1+y / \log x)$ implique alors, dans les hypothèses effectuées,

$$
\alpha \bar{u} \gg \log \left(1+\frac{y}{\log x}\right) \frac{\min (y, \log x)}{(\log y)^{2}} \gg \min \left(h(x), \frac{\log x}{\left(\log _{2} x\right)^{2}}\right),
$$

d'où $(5 \cdot 17)$.

Lorsque $u \leqslant \sqrt{\log y}$, et donc $\mathrm{e}^{(\log x)^{2 / 3}} \leqslant y \leqslant x^{1 / h(x)}$, nous pouvons faire appel au corollaire 5.2 de [3] qui fournit directement $(5 \cdot 17)$.

Dans l'énoncé suivant, nous posons

$$
\begin{aligned}
& E:=\frac{(y+\log x) \log y}{x^{\alpha} y} \ll \frac{\log x}{\pi(y)}\left(1+\frac{\vartheta(y)}{\log x}\right)^{1-u}, \\
& F:=\frac{(y+\log x) \sqrt{\bar{u}+\log _{2} y}}{x^{\alpha} \bar{u} \sqrt{\log x}} \ll \frac{\sqrt{\log x} \sqrt{\bar{u}+\log _{2} y}}{\bar{u} y}\left(1+\frac{\vartheta(y)}{\log x}\right)^{1-u} .
\end{aligned}
$$

où nous avons fait appel à (1-13).

Lemme 5.4. Uniformément pour $x \geqslant y \geqslant 2$ et $f \in \mathbb{A}_{0}$, nous avons

$$
\frac{V_{f}(x, y)}{\mathbb{V}\left(Z_{f}\right)}=\frac{V_{f}^{*}(x, y)}{\mathbb{V}\left(Z_{f}^{*}\right)}\{1+O(E)\}+O(F) .
$$

En particulier, pour tout $\varepsilon>0$, nous avons, lorsque $x \rightarrow \infty$ et $y \geqslant(1+\varepsilon) \log _{2} x \log _{3} x$,

$$
\frac{V_{f}(x, y)}{\mathbb{V}\left(Z_{f}\right)}=\frac{V_{f}^{*}(x, y)}{\mathbb{V}\left(Z_{f}^{*}\right)}\{1+o(1)\}+o(1) .
$$

De plus, dans le domaine $2 \leqslant y \leqslant \log x$, nous avons

$$
V_{f}(x, y)-V_{f}^{*}(x, y) \ll \mathbb{V}\left(Z_{f}\right)\left(\frac{y^{9 / 2}}{x^{\alpha / 2}(\log x)^{3 / 2}}+\frac{y}{x^{\alpha} \log y}\right) .
$$

Remarques. (i) Compte tenu de (5·7), nous pouvons déduire de $(5 \cdot 19)$ que, pour tout $u \geqslant 1$ fixé,

$$
\limsup _{x \rightarrow \infty} \sup _{f \in \mathbb{A}_{0}} \frac{V_{f}^{*}\left(x, x^{1 / u}\right)}{\mathbb{V}\left(Z_{f}^{*}\right)}=\lambda(u) .
$$

(ii) La minoration de $x^{\alpha}$ indiquée en (1·13), implique l'existence d'une constante positive convenable $c$ telle que l'on ait $x^{\alpha} \gg \mathrm{e}^{c y / \log y}$ dans le domaine $2 \leqslant y \leqslant \log x$. Cela permet de remplacer le membre de droite de (5.20) par $\mathbb{V}\left(Z_{f}\right) \mathrm{e}^{-c_{1} y / \log y}$ avec $c_{1}>0$.

2. En effet, la formule (2.1) de [4] s'écrit, avec les notations de [4],

$$
V_{f}(x, y)=\mathbb{V}\left(Z_{f}\right)+T_{f}(x, y)+V_{f}^{*}(x, y)-U_{f}(x, y)
$$

où, d'après la dernière formule centrée de l'article, $U_{f}(x, y) \ll B^{2}(x, y) / \bar{u}$.

L'inégalité $T_{f}(x, y) \leqslant C_{1} B^{2}(x, y) / \bar{u}$, où $C_{1}$ est une constante absolue, est établie au milieu de la page 263.

Enfin, la formule (2.4) de [4] implique $V_{f}^{*}(x, y)=S^{*}+O\left(B^{2}(x, y) / \bar{u}\right)$ où $S^{*} \leqslant C_{2} B^{2}(x, y) / \bar{u}$ et

$C_{2}$ désigne une constante absolue. 
Démonstration. Nous avons vu en $(3 \cdot 12)$ que

$$
V_{f}(x, y)-V_{f}^{*}(x, y)=\left\{\mathbb{E}\left(Z_{f}^{*}\right)-\mathbb{E}\left(Z_{f}\right)\right\}\left\{2\left(M_{1}-\mathbb{E}\left(Z_{f}^{*}\right)\right)+\mathbb{E}\left(Z_{f}\right)-\mathbb{E}\left(Z_{f}^{*}\right)\right\} .
$$

Compte tenu de la majoration $(3 \cdot 6)$, nous pouvons écrire

$$
\left\{M_{1}-\mathbb{E}\left(Z_{f}^{*}\right)\right\}^{2}=\left\{\sum_{p \leqslant y} f(p) r_{p}\right\}^{2} \ll \frac{1}{\bar{u}^{2}} \sum_{p \leqslant y} \frac{f(p)^{2} g_{p}(\alpha)}{p^{\alpha}} \sum_{p \leqslant y} \frac{g_{p}(\alpha)}{p^{\alpha}} \ll \mathbb{V}\left(Z_{f}^{*}\right) \frac{\bar{u}+\log _{2} y}{\bar{u}^{2}},
$$

où la dernière somme en $p$ a été évaluée comme indiqué au lemme 3.2 de [3]. La majoration $w_{p} \ll(p / x)^{\alpha}$ mentionnée en $(1 \cdot 12)$ fournit de plus

$$
\begin{aligned}
\left\{\mathbb{E}\left(Z_{f}\right)-\mathbb{E}\left(Z_{f}^{*}\right)\right\}^{2} & =\left\{\sum_{p \leqslant y} f(p) \frac{w_{p}}{p^{\alpha}}\right\}^{2} \leqslant \frac{1}{x^{2 \alpha}} \sum_{p \leqslant y} \frac{f(p)^{2} g_{p}(\alpha)}{p^{\alpha}} \sum_{p \leqslant y} \frac{p^{\alpha}}{g_{p}(\alpha)} \\
& \ll \frac{\pi(y) y^{\alpha}}{x^{2 \alpha}}\left(\frac{1}{\alpha \log y}+1\right) \mathbb{V}\left(Z_{f}^{*}\right) \ll \frac{y^{\alpha}\{u+\pi(y)\}}{x^{2 \alpha}} \mathbb{V}\left(Z_{f}^{*}\right) .
\end{aligned}
$$

Or, d'après les formules (7.6) et (7.8) de [7], nous avons

$$
y^{\alpha} \ll\left(1+\frac{y}{\log x}\right) \log y \ll \frac{y+\log x}{u},
$$

donc

$$
\left\{\mathbb{E}\left(Z_{f}\right)-\mathbb{E}\left(Z_{f}^{*}\right)\right\}^{2} \ll \mathbb{V}\left(Z_{f}^{*}\right) \frac{(y+\log x)^{2}}{x^{2 \alpha} \log x}
$$

Il s'ensuit que

$$
V_{f}(x, y)-V_{f}^{*}(x, y) \ll \mathbb{V}\left(Z_{f}^{*}\right) \frac{(y+\log x) \sqrt{\left(\bar{u}+\log _{2} y\right)}}{x^{\alpha} \bar{u} \sqrt{\log x}}=\mathbb{V}\left(Z_{f}^{*}\right) F .
$$

Par ailleurs, la majoration

$$
\frac{w_{p}}{p^{\alpha} g_{p}(\alpha)} \ll \frac{1}{x^{\alpha} \alpha} \ll \frac{(\log x+y) \log y}{x^{\alpha} y},
$$

implique

$$
\mathbb{V}\left(Z_{f}^{*}\right)-\mathbb{V}\left(Z_{f}\right)=\sum_{p \leqslant y} \frac{f(p)^{2} w_{p}}{p^{2 \alpha}} \ll \mathbb{V}\left(Z_{f}^{*}\right) \frac{(y+\log x) \log y}{x^{\alpha} y}=\mathbb{V}\left(Z_{f}^{*}\right) E .
$$

Compte tenu de l'identité

$$
\frac{V_{f}(x, y)}{\mathbb{V}\left(Z_{f}\right)}\left(1+\frac{\mathbb{V}\left(Z_{f}\right)-\mathbb{V}\left(Z_{f}^{*}\right)}{\mathbb{V}\left(Z_{f}^{*}\right)}\right)-\frac{V_{f}^{*}(x, y)}{\mathbb{V}\left(Z_{f}^{*}\right)}=\frac{V_{f}(x, y)-V_{f}^{*}(x, y)}{\mathbb{V}\left(Z_{f}^{*}\right)} .
$$

les majorations $(5 \cdot 23)$ et $(5 \cdot 24)$ impliquent bien l'estimation $(5 \cdot 18)$ annoncée.

Pour établir (5.19), nous observons que, sous les hypothèses effectuées, nous avons certainement

$$
\begin{aligned}
& E \ll(\log x) \mathrm{e}^{-(1-\varepsilon / 3) y / \log y} \ll(\log x)^{-\varepsilon(1-\varepsilon) / 2}, \\
& F \ll \sqrt{\log x}\left(1+\frac{\vartheta(y)}{\log x}\right)^{-u} \ll(\log x)^{-(1-\varepsilon) / 2} .
\end{aligned}
$$


Il reste à établir $(5 \cdot 20)$ dans le domaine $(3 \cdot 7)$. D'après $(3 \cdot 10)$ et $(5 \cdot 12)$, nous avons sous cette hypothèse

$$
M_{1}-\mathbb{E}\left(Z_{f}^{*}\right)=\sum_{p \leqslant y} f(p) r_{p} \ll \sum_{p \leqslant y} g_{p}(\alpha) \frac{|f(p)|}{p^{\alpha}}\left(\frac{1}{u}+\frac{y^{2} \pi(y)}{(\log x)^{2}}\right),
$$

d'où

$$
\begin{aligned}
\left\{M_{1}-\mathbb{E}\left(Z_{f}^{*}\right)\right\}^{2} & \ll \mathbb{V}\left(Z_{f}^{*}\right) \sum_{p \leqslant y} \frac{g_{p}(\alpha)}{p^{\alpha}}\left(\frac{1}{u}+\frac{y^{2} \pi(y)}{(\log x)^{2}}\right)^{2} \\
& \ll \mathbb{V}\left(Z_{f}^{*}\right)\left\{\frac{y^{2} \log y}{(\log x)^{3}}+\frac{y^{5} \pi(y)^{3}}{(\log x)^{5}}\right\} .
\end{aligned}
$$

En reportant dans $(5 \cdot 22)$ les majorations $(3 \cdot 9)$ et $(5 \cdot 25)$, nous obtenons bien $(5 \cdot 20)$ sous les hypothèses effectuées.

\subsection{Démonstration du Théorème 5.1.}

Commençons par établir l'assertion (iv). En reportant (5.9) et (5.10) dans (3.13), nous obtenons bien $(5 \cdot 6)$ dans le domaine $(3 \cdot 7)$. Nous observons incidemment que $(5 \cdot 2)$ en découle dans le sous-domaine $y=o(\log x)$.

Considérons ensuite le point (iii). Selon l'hypothèse effectuée, il existe une fonction $h(x) \rightarrow \infty$ telle que

$$
h(x) \leqslant y \leqslant(\log x) / h(x) .
$$

Nous avons alors $V_{f}^{*}(x, y)=\mathbb{V}\left(Z_{f}^{*}\right)\{1+o(1)\}$ d'après (5·6). En reportant cette inégalité dans $(5 \cdot 20)$, nous obtenons

$$
V_{f}(x, y)=\mathbb{V}\left(Z_{f}^{*}\right)+o\left(\mathbb{V}\left(Z_{f}\right)\right)
$$

Or, en comparant les identités données en $(3 \cdot 8)$ pour $\mathbb{V}\left(Z_{f}\right)$ et en $(3 \cdot 14)$ pour $\mathbb{V}\left(Z_{f}^{*}\right)$, nous obtenons, lorsque $y$ tend vers l'infini,

$$
\frac{\mathbb{V}\left(Z_{f}\right)}{\mathbb{V}\left(Z_{f}^{*}\right)} \leqslant 1+\max _{p \leqslant y}\left(\frac{p^{\alpha}}{x^{\alpha} g_{p}(\alpha)}\right)=1+\frac{1+o(1)}{x^{\alpha} \alpha \log 2}
$$

et

$$
\frac{\mathbb{V}\left(Z_{f}\right)}{\mathbb{V}\left(Z_{f}^{*}\right)} \geqslant\{1+o(1)\} \min _{p \leqslant y}\left(1+\frac{p^{\alpha}}{x^{\alpha} g_{p}(\alpha)}\right)=\{1+o(1)\} \frac{1+x^{\alpha} \alpha \log y}{x^{\alpha} \alpha \log y} .
$$

L'estimation (5·15) de $\alpha$ fournit bien (5.5) dans le domaine (5·26).

Pour établir l'assertion (ii), i.e. (5·4) dans le domaine

$$
h(x) \leqslant y \leqslant x^{1 / h(x)}
$$

dès que $h(x) \rightarrow \infty$, nous observons d'abord que la conclusion souhaitée découle de $(5 \cdot 5)$ sous la condition $(5 \cdot 26)$. Comme le Lemme 5.3 fournit $(5 \cdot 4)$ sous la condition $(5 \cdot 16)$, nous obtenons bien $(5 \cdot 4)$ dans le domaine $(5 \cdot 28)$ quitte à choisir $h(x) \leqslant \log _{2} x$ dans $(5 \cdot 26)$ et $(5 \cdot 16)$.

Il reste à prouver l'assertion (i). En vertu de (5·6), l'inégalité requise $(5 \cdot 2)$ est en fait une égalité sous la condition $y=o(\log x)$. Nous pouvons donc supposer

$$
(\log x) / h(x) \leqslant y \leqslant x^{1 / h(x)},
$$

où $h(x)$ est une fonction qui tend vers $+\infty$. Or, dans ce domaine, la relation $(5 \cdot 19)$ implique que, si le rapport $V_{f}(x, y) / \mathbb{V}\left(Z_{f}\right)$ est $\leqslant 1+o(1)$, alors il en va de même pour $V_{f}^{*}(x, y) / \mathbb{V}\left(Z_{f}^{*}\right)$. L'assertion (ii) fournit donc le résultat requis. 


\subsection{Le cas $f=\omega$}

Établissons ici $(5 \cdot 8)$ sous l'hypothèse que $y$ et $(\log x) / y$ tendent vers l'infini.

D'après $(3 \cdot 2)$ et $(1 \cdot 20)$, nous avons

$$
\mathbb{V}\left(Z_{\omega}\right)=\sum_{p \leqslant y} \frac{1-w_{p}}{p^{\alpha}}\left(g_{p}(\alpha)+\frac{w_{p}}{p^{\alpha}}\right), \quad \mathbb{V}\left(Z_{\omega}^{*}\right)=\sum_{p \leqslant y} \frac{g_{p}(\alpha)}{p^{\alpha}}
$$

Comme (5.15) implique, dans la circonstance considérée, que l'on a $w_{p}=o(1)$ et $p^{\alpha}=1+o(1)$ uniformément pour $p \leqslant y$, il s'ensuit que

$$
\mathbb{V}\left(Z_{\omega}\right)-\mathbb{V}\left(Z_{\omega}^{*}\right)=\{1+o(1)\} \sum_{p \leqslant y} \frac{w_{p}}{p^{\alpha}}+o\left(\mathbb{V}\left(Z_{\omega}^{*}\right)\right)
$$

Si, par exemple, $y>\left(\log _{2} x\right)^{2}$, alors $\sup _{p \leqslant y} w_{p} / g_{p}(\alpha)=o(1)$. Nous obtenons donc $V_{\omega} \sim \mathbb{V}\left(Z_{\omega}^{*}\right)$, et $(5 \cdot 8)$ résulte de $(5 \cdot 27)$.

Dans le cas contraire, nous avons $\alpha \log y=o(1)$ et $\alpha \log x=\pi(y)+o(1)$ en vertu de $(5 \cdot 15)$, donc $w_{p} / p^{\alpha} \sim \mathrm{e}^{-\pi(y)}$ uniformément pour $p \leqslant y$. Il vient donc

$$
\sum_{p \leqslant y} \frac{w_{p}}{p^{\alpha}} \sim \pi(y) \mathrm{e}^{-\pi(y)}
$$

alors que

$$
\mathbb{V}\left(Z_{\omega}^{*}\right) \sim \alpha \vartheta(y) \sim \frac{y \pi(y)}{\log x} .
$$

Par (5·30), nous en déduisons que

$$
\mathbb{V}\left(Z_{\omega}\right) \sim\left\{1+\frac{\log x}{y \mathrm{e}^{\pi(y)}}\right\} \mathbb{V}\left(Z_{\omega}^{*}\right) .
$$

En reportant dans $(5 \cdot 27)$, nous obtenons à nouveau $(5 \cdot 8)$.

\subsection{Démonstration du Théorème 2.1}

Soit $f$ une fonction arithmétique additive. On peut écrire, lorsque $n \in S(x, y)$,

$$
f(n)-\mathbb{E}\left(Z_{f}^{*}\right)=\sum_{p \leqslant y} c_{n, p} y_{p}
$$

avec $y_{p}:=f(p) \sqrt{g_{p}(\alpha) / p^{\alpha}}$ et

$$
\begin{cases}c_{n, p}:=\sqrt{p^{\alpha} / g_{p}(\alpha)}-\sqrt{g_{p}(\alpha) / p^{\alpha}} & \text { si } p \mid n, \\ c_{n, p}:=-\sqrt{g_{p}(\alpha) / p^{\alpha}} & \text { si } p \nmid n .\end{cases}
$$

La majoration (1·19) équivaut donc à la validité de

$$
\sum_{n \in S(x, y)}\left|\sum_{p \leqslant y} c_{n, p} y_{p}\right|^{2} \leqslant C \Psi(x, y) \sum_{p \leqslant y}\left|y_{p}\right|^{2}
$$

pour tout choix des nombres complexes $y_{p}$. Le principe de dualité exprimant l'égalité des normes d'un opérateur d'espaces de Hilbert et de son adjoint (cf., par exemple, le lemme I.4.5.1 de [9]) montre alors que cette majoration est équivalente à la validité de l'inégalité

$$
\sum_{p \leqslant y}\left|\sum_{n \in S(x, y)} c_{n, p} a_{n}\right|^{2} \leqslant C \Psi(x, y) \sum_{n \in S(x, y)}\left|a_{n}\right|^{2}
$$

pour toute suite $\left\{a_{n}\right\}_{n=1}^{\infty}$ de nombres complexes. 


\section{La répartition des facteurs premiers d'un entier friable}

\subsection{Preuve du Théorème 2.2}

Posons

$$
W_{t}:=\mathbb{V}\left(Z_{\omega_{t}}^{*}\right)=\sum_{p \leqslant t} \frac{g_{p}(\alpha)}{p^{\alpha}} \ll \min (1, \alpha \log t) M_{t} \quad(2 \leqslant t \leqslant y \leqslant x) .
$$

Il résulte du Théorème 1.2 que, pour chaque $t$ fixé dans $[2, y], y \leqslant \log x$,

$$
\sum_{\substack{n \in S(x, y) \\(n)-M_{t} \mid>h \sqrt{W_{t}}}} 1 \ll \frac{\Psi(x, y)}{h^{2}} \quad(x \geqslant y \geqslant 2, h>0) .
$$

Observons d'emblée que, si $\alpha=o(1)$, alors, pour tout entier $T=T(x, y)$ tel que $\alpha T=o(1)$, nous pouvons choisir, par exemple, $h:=(\alpha T)^{-1 / 4}$ pour obtenir $\omega_{T}(n)=M_{T}+o(1)$ pour tous les entiers $n$ de $S(x, y)$ sauf au plus $o(\Psi(x, y))$ d'entre eux. Comme $M_{T}=\pi(T)+O(\alpha T)$, cela implique en fait $\omega_{T}(n)=\pi(T)$, avec le même nombre d'exceptions.

Pour traiter le cas général, nous introduisons une constante positive $c$ assez petite, rappelons la notation $z:=\min \{y, \exp (1 / \alpha)\}$ et, dans un premier temps, introduisons l'ensemble $\mathcal{K}(x, y)$ des entiers naturels $k$ tels que

$$
M_{2} \leqslant c \alpha k^{3}(\log k)^{1+b} \leqslant M_{z}
$$

Pour tout $k$ de $\mathcal{K}(x, y)$, il existe alors un $t_{k} \in[2, z]$ tel que

$$
M_{t_{k}} \leqslant c \alpha k^{3}(\log k)^{1+b} \leqslant M_{t_{k}}+1 \text {. }
$$

Cela résulte du fait que la fonction $t \mapsto M_{t}$ croît par sauts n'excédant pas l'unité.

Pour tous les entiers $k$ de $\mathcal{K}(x, y)$, nous avons $W_{t_{k}} \ll \alpha M_{t_{k}} \log t_{k}$ et, en vertu de $(2 \cdot 2)$, $M_{t_{k}} \asymp t_{k} / \log t_{k}$, donc $t_{k} \ll \alpha k^{3}(\log k)^{b+2}$. En spécialisant $t=t_{k}$ et $h \asymp k^{1 / 2}(\log k)^{b / 2}$ dans $(6 \cdot 1)$, nous obtenons que l'estimation

$$
\left|\omega_{t_{k}}(n)-M_{t_{k}}\right| \leqslant c^{2} \alpha k^{2}(\log k)^{1+b} \leqslant c \alpha^{1 / 3} M_{t_{k}}^{2 / 3}\left\{\log \left(3 M_{t_{k}} / \alpha\right)\right\}^{2 b / 3}
$$

a lieu, avec un choix convenable de $c$, pour tous les entiers $n$ de $S(x, y)$ sauf peut-être pour au plus $\ll \Psi(x, y) /\left\{k(\log k)^{b}\right\}$ d'entre eux. Comme

$$
M_{t_{k+1}}-M_{t_{k}} \leqslant c^{3 / 4} \alpha k^{2}(\log k)^{1+b}+1 \leqslant c^{1 / 12} \alpha^{1 / 3} M_{t_{k}}^{2 / 3}\left\{\log \left(3 M_{t_{k}} / \alpha\right)\right\}^{2 b / 3}+1,
$$

et compte tenu la croissance en $t$ de $\omega_{t}(n)$, il s'ensuit, par sommation sur $k$, que nous obtenons bien la validité de la première estimation $(2 \cdot 3)$ dans les conditions de l'énoncé.

Dans le cas $z<t \leqslant y$, et donc $\alpha>1 / \log y$, l'inégalité (2·3) a été établie au théorème 1.6 de [3]. Pour la commodité du lecteur, rappelons rapidement les détails. Nous introduisons des points-tests $\left.\left.s_{k} \in\right] z, y\right]$ tels que $M_{s_{k}} \leqslant c k^{3}(\log k)^{b}<M_{s_{k}}+1$. Pour le même choix de $h$ que précédemment dans $(6 \cdot 1)$, nous obtenons alors que

$$
\omega_{s_{k}}(n)-M_{s_{k}} \ll c^{1 / 3} M_{s_{k}}^{2 / 3}\left(\log M_{s_{k}}\right)^{b / 3}
$$

pour toutes les valeurs admissibles de $k$. Cela implique bien le résultat annoncé. 


\section{2. Preuve du Corollaire 2.3}

Appliquons $(2 \cdot 3)$ avec $t=p_{j}(n)$ et donc $\omega_{t}(n)=j$. Compte tenu de l'approximation $M_{t}-\pi(t) \ll \alpha t \ll \pi(t)$, la première majoration fournit $p_{j}(n) \leqslant \mathrm{e}^{1 / \alpha}$ sous l'hypothèse $p_{j} \leqslant \mathrm{e}^{1 / 2 \alpha}$ dès que $x$, et donc $J_{x}$, est assez grand. Il suit

$$
\pi\left(p_{j}(n)\right)-j=\pi\left(p_{j}(n)\right)-\pi\left(p_{j}\right) \ll \alpha^{1 / 3} j^{2 / 3}\{\log (3 j / \alpha)\}^{2 b / 3}+1 .
$$

Cela implique $\log p_{j}(n) \asymp \log p_{j}$ et donc

$$
p_{j}(n)-p_{j} \ll\left\{\pi\left(p_{j}(n)\right)-\pi\left(p_{j}\right)\right\} \log p_{j} .
$$

On en déduit immédiatement la première estimation de $(2 \cdot 5)$.

Observons par ailleurs que $(2 \cdot 3)$ implique

$$
\omega_{t}(n)-M_{t} \ll M_{t}^{2 / 3}\left(\log M_{t}\right)^{2 b / 3} \quad(2 \leqslant t \leqslant y) .
$$

En spécialisant $t=p_{j}(n)$ et en tenant compte de $(2 \cdot 2)$, nous obtenons, pour $j$ assez grand,

$$
\log w_{j}+\frac{\mathrm{e}^{w_{j}}-1}{w_{j}}+O\left(\frac{\mathrm{e}^{w_{j}}-1}{w_{j}^{2}}\right)=j
$$

où l'on a posé $w_{j}:=(1-\alpha) \log p_{j}(n)$. Sous l'hypothèse $p_{j}>\mathrm{e}^{1 / 2 \alpha}$, et donc $\alpha \log j \gg 1$, le dernier terme d'erreur est $\ll \alpha j$. En inversant le terme principal du membre de gauche de $(6 \cdot 3)$ comme indiqué dans [3], nous obtenons,

$$
w_{j}=\log (j \log j)+\frac{\log _{2} j}{\log j}+O(\alpha)=\log p_{j}+O(\alpha),
$$

où nous avons fait appel à l'estimation classique $p_{j}=j \log (j \log j)+O(j)$. Cela implique la seconde estimation de $(2 \cdot 5)$.

La précision finale de l'énoncé découle immédiatement de la dernière assertion du Théorème 2.2 .

\section{Bibliographie}

[1] K. Alladi, The Turán-Kubilius inequality for integers without large prime factors, J. reine angew. Math. 335 (1982), 180-196.

[2] R. de la Bretèche \& G. Tenenbaum, Propriétés statistiques des entiers friables, Ramanujan J. 9 (2005), 139-202.

[3] R. de la Bretèche \& G. Tenenbaum, Entiers friables : inégalité de Turán-Kubilius et applications, Invent. Math. 159 (2005), 531-588.

[4] R. de la Bretèche \& G. Tenenbaum, On the friable Turán-Kubilius inequality, Proceedings of the Fifth International Conference Analytic and Probabilistic Methods in Number Theory held on the occasion of Professor Jonas Kubilius' 90th birthday (2012), E. Manstavičius et al. (eds), TEV Vilnius 2012, 259-265.

[5] R. de la Bretèche \& G. Tenenbaum, Entiers friables et méthode des résidus, prépublication (2014).

[6] G. Hanrot, B. Martin \& G. Tenenbaum, Constantes de Turán-Kubilius friables : étude numérique, Exp. Math. Experiment. Math. 19, $\mathbf{n}^{\circ} 3$ (2010), 345-361.

[7] A. Hildebrand \& G. Tenenbaum, On integers free of large prime factors, Trans. Amer. Math. Soc. 296 (1986), no 1, 265-290.

[8] B. Martin \& G. Tenenbaum, Sur l'inégalité de Turán-Kubilius friable, J. reine angew. Math.647 (2010), 175-234.

[9] G. Tenenbaum, Introduction à la théorie analytique et probabiliste des nombres, troisième édition, coll. Échelles, Belin, 2008, 592 pp.

[10] T.Z. Xuan, The Turán-Kubilius inequality for integers free of large prime factors, J. Number Theory 43 (1993), 82-87.

[11] T.Z. Xuan, The Turán-Kubilius inequality for integers free of large prime factors (II), Acta Arith. 65 (1993), 329-352. 
Université Paris Diderot-Paris 7

Sorbonne Paris Cité, UMR 7586

Institut de Mathématiques de Jussieu-PRG

Case 7012, F-75013 Paris

France

regis.delabreteche@imj-prg.fr
Gérald Tenenbaum

Institut Elie Cartan

Université de Lorraine

BP 70239

F-54506 Vandœuvre-lès-Nancy Cedex

France

gerald.tenenbaum@univ-lorraine.fr 